\title{
Entwicklung der alpinen Abflussregime in Österreich im Zeitraum 1961-2010
}

\author{
Katharina Lebiedzinski · Josef Fürst (iD
}

Online publiziert: 2. Juli 2018

(C) Der/die Autor(en) 2018

Zusammenfassung Die saisonale Abflusscharakteristik im Alpenraum hat sich im Lauf der letzten Jahrzehnte gewandelt. Die vorliegende Studie widmet sich der Untersuchung räumlicher und zeitlicher Entwicklungen von Abflussregimen. Diese Entwicklungen wurden österreichweit im Bezugszeitraum 1961-2010 an 280 anthropogen unbeeinflussten Stationen analysiert. Mittels einer Clusteranalyse wurden fünf charakteristische Regimegruppen bestimmt, die durch glaziale, nivale, pluviale sowie nivo-pluviale Merkmale geprägt sind. Ihre räumliche Verteilung zeigt trotz der topografischen und hydrologischen Heterogenität Österreichs ein deutliches Muster.

Der Alpenraum wird durch nivale und glaziale Abflussregime dominiert. Bei Regimen des Flach- und Hügellandes dominierten hingegen pluviale Merkmale. In der zeitlichen Entwicklung nahmen pluviale Einflüsse in weiten Teilen signifikant $\mathrm{zu}$, die in Nord-Süd-Richtung unterschiedliche Reaktionen in der mittleren saisona-

\author{
Zusatzmaterial online Zusätzliche \\ Informationen sind in der \\ Online-Version dieses Artikels \\ (https://doi.org/10.1007/s00506- \\ 018-0499-z) enthalten. Das \\ Onlinematerial umfasst eine \\ kartographische Darstellung der \\ zeitlichen Entwicklung saisonaler \\ Abflusscharakteristiken und ihrer \\ räumlichen Verteilung. $\mathrm{Zu}$ sehen sind \\ gleitende 10-Jahres-Mittelwerte der \\ Pardé-Koeffizienten für den Zeitraum \\ 1961-2010 an allen untersuchten \\ Stationen in Österreich. \\ DI K. Lebiedzinski . \\ ao. Univ.-Prof. DI Dr. J. Fürst ( $ه)$ \\ Institut für Wasserwirtschaft, \\ Hydrologie und konstruktiven \\ Wasserbau (IWHW), Universität \\ für Bodenkultur Wien, \\ Muthgasse 18, 1190 Wien, Österreich
}

josef.fuerst@boku.ac.at len Abflusscharakteristik hervorgerufen haben.

Die beobachteten Veränderungen der Abflussregime sind konsistent mit den saisonalen Trends im Niederschlag, Abfluss und der Wasserbilanz des Hydrologischen Atlas Österreichs. Der ermittelte Stand mittlerer saisonaler Abflusscharakteristiken und seine bisherige Entwicklung ist eine wichtige Informationsquelle angesichts der prognostizierten Veränderungen der Steuerfaktoren abflussbildender Prozesse durch den Klimawandel.

\section{Development of alpine flow regimes in Austria in the period 1961-2010}

Abstract The seasonal runoff characteristics in Alpine rivers have changed during the last decades. The present study deals with the investigation of temporal changes and spatial distribution of seasonal flow characteristics by identifying and describing flow regimes. Mean monthly flows at 280 stream gauges without significant anthropogenic influences in Austria were analyzed for the period 1961-2010. A cluster analysis was used to define five representative flow regime groups with glacial, nival, pluvial and nivopluvial characteristics, respectively. Although both topographic and hydrological conditions in Austria are highly heterogeneous, the spatial distribution shows a clear pattern.

The alpine region was dominated by nival and glacial flow regimes, while the lowland and hilly region in the northeast was dominated by pluvial flow regimes. The temporal development showed a significant increase of pluvial characteristics over time, responding differently at mean seasonal flows north and south of the Alpine divide.

The observed changes in the flow regimes were consistent with seasonal trends in precipitation, discharge and the water balance as published in the Hydrologic Atlas of Austria. The study provides new information about the temporal development and the current state of mean seasonal flow characteristics, that is of particular interest considering predicted changes of factors and processes influencing flow generation.

\section{Einleitung}

Der Abfluss von Fließgewässern ist im zeitlichen Verlauf durch die aktuellen und rezenten meteorologischen Verhältnisse geprägt, insbesondere Niederschlag und Temperatur, weist aber auch einen klimatisch und geografisch bedingten charakteristischen Jahresgang auf.

Die Alpen haben einen Anteil von $65 \%$ an der Gesamtfläche Österreichs, und daher sind die Abflüsse stark durch alpine Einflüsse geprägt. Der Klimawandel und seine Folgen (APCC 2014; Fürst et al. 2010) sowie anthropogene Eingriffe in den Wasserhaushalt von Fließgewässern (Poff et al. 1997) sind bestimmende Faktoren von hoher Aktualität, die auch die Abflussverhältnisse beeinflussen. Eine sich im Wandel befindliche Umwelt beeinflusst langjährige, standorttypische Abflusscharakteristika und gegebenenfalls auch die Wasserverfügbarkeit für die menschliche Nutzung. Daher ist die Kenntnis des langjährigen, charakteristischen Abflussverhaltens und ebenso seiner Veränderungen von zentraler Bedeutung für verschiedenste Fragestellungen z.B. in der Schutzund Energiewasserwirtschaft, Ökologie und im Tourismus (Holzmann und Koboltschnig 2010; Jungwirth et al. 2003; Mader et al. 1996).

Das charakteristische Abflussverhalten eines Fließgewässers einer bestimmten Region kann mittels Abflussregimen beschrieben werden. Sie klassifizieren Fließgewässer anhand hydrologischer Merkmale und geben die 


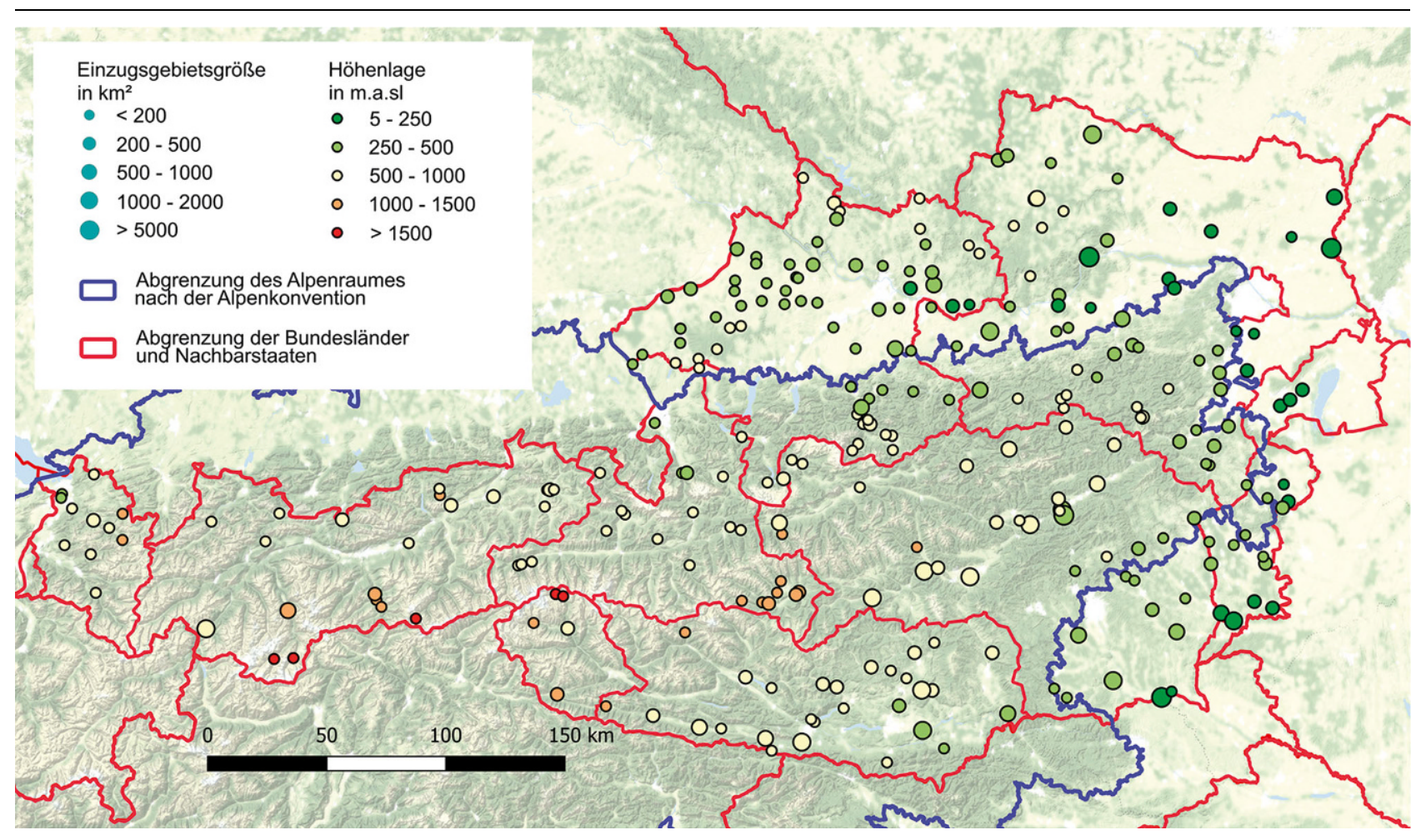

Abb. 1 Übersicht über die Abflussmessstellen in Abhängigkeit von Höhenlage (Symbolfarbe) und Einzugsgebietsgröße (Symbolgröße)

charakteristische saisonale Verteilung des Abflusses im Betrachtungszeitraum eines Jahres wieder. Das Abflussregime kann allerdings auch als Indikator von räumlichen und zeitlichen Abflussveränderungen betrachtet werden und daher zur Bestimmung gegenwärtiger sowie zukünftiger Veränderungen herangezogen werden (Wrzesinski und Sobkowiak 2018). Je nach zu untersuchender hydrologischer Kenngröße, wird zwischen den Teilregimen Mittelwasser-, Hochwasser- und Niederwasserregime unterschieden, die eine unterschiedliche Bedeutung für wasserwirtschaftliche und ökologische Aufgaben aufweisen (z.B. Restwasserabgabe, Speicherbewirtschaftung) (Pfaundler et al. 2011). Für Mittelwasserregime stellen mittlere monatliche und mittlere jährliche Abflüsse die grundlegenden hydrologischen Kenngrößen dar, für Hochwasserregime sind es mittlere jährliche Hochwasserabflüsse, die Hochwasserhäufigkeit sowie -saisonalität und schließlich für Niederwasserregime die Niederwasserabflüsse, ihre Saisonalität sowie die Dauer von Niederwasserperioden.

Die Abflussregimeforschung ist trotz jahrzehntelanger Forschungsgeschichte immer noch aktuell. Wurde in den
Anfängen das Abflussverhalten ausgewählter großer Flüsse untersucht (Pardé 1933; Grimm 1968), steht nun immer häufiger die vergangene wie auch zukünftige Entwicklung der unterschiedlichen Teilregimetypen und der Zusammenhang mit klimatischen sowie anthropogenen Veränderungen im Vordergrund (Wilson et al. 2013; Bower et al. 2004). Auf europäischer Ebene gaben Stahl et al. (2010) einen groben Überblick über Veränderungen von Mittelwasserregimen und Trends im Abfluss an ausgewählten unbeeinflussten Stationen, um Einflüsse des Klimawandels zu ermitteln. Die untersuchten Stationen zeigten in weiten Teilen positive Trends im Jahresabfluss, davon ausgenommen waren die südlichen und östlichen Regionen Europas. Die Mittelwasserregime wiesen zumeist positive Trends in den Wintermonaten auf, während von April bis August negative Trends auftraten.

Ebenfalls auf europäischer Ebene gelang es Hall et al. (2014) mit einer Literaturstudie, den Status quo der Hochwasserregime und ihrer Analyseverfahren zu untersuchen. Generell zeigten Hochwässer in den nördlichen, östlichen sowie südwestlichen Regionen negative Trends. Diese sind auf Ver- änderungen der Schneeschmelze bzw. der Bodenfeuchte zurückzuführen. Im Westen und in Zentraleuropa (inklusive Alpenraum) sind positive Trends aufgetreten, die im Zusammenhang mit Veränderungen im Niederschlag stehen. Parajka et al. (2010) legten in einer überregionalen Studie zur Analyse der Saisonalität von Hochwasserregimen den Fokus auf die Alpen und Karpaten. Demnach treten Extremhochwässer tendenziell zumeist im Herbst auf, während kleinere Hochwasserereignisse im Sommer $\mathrm{zu}$ erwarten sind. Für ausgewählte anthropogen unbeeinflusste Einzugsgebiete in Österreich haben Laaha et al. (2016) die Auswirkungen auf Niederwasserregime untersucht. Von besonderer Bedeutung sind hier die Gefrier- und Schmelzprozesse im Winter und die Verdunstung im Sommer.

Da die Alpenregion aus hydrologischer Sicht aufgrund nival und glazial geprägter Merkmale einen besonderen Stellenwert einnimmt, wurden in unterschiedlichen Studien die Auswirkungen des Klimawandels auf zukünftige Abflussregime in alpinen Regionen untersucht (Bard et al. 2015; Milano et al. 2015; Zampieri et al. 2015; Laghari et al. 2012; Vanham und Rauch 2009). 


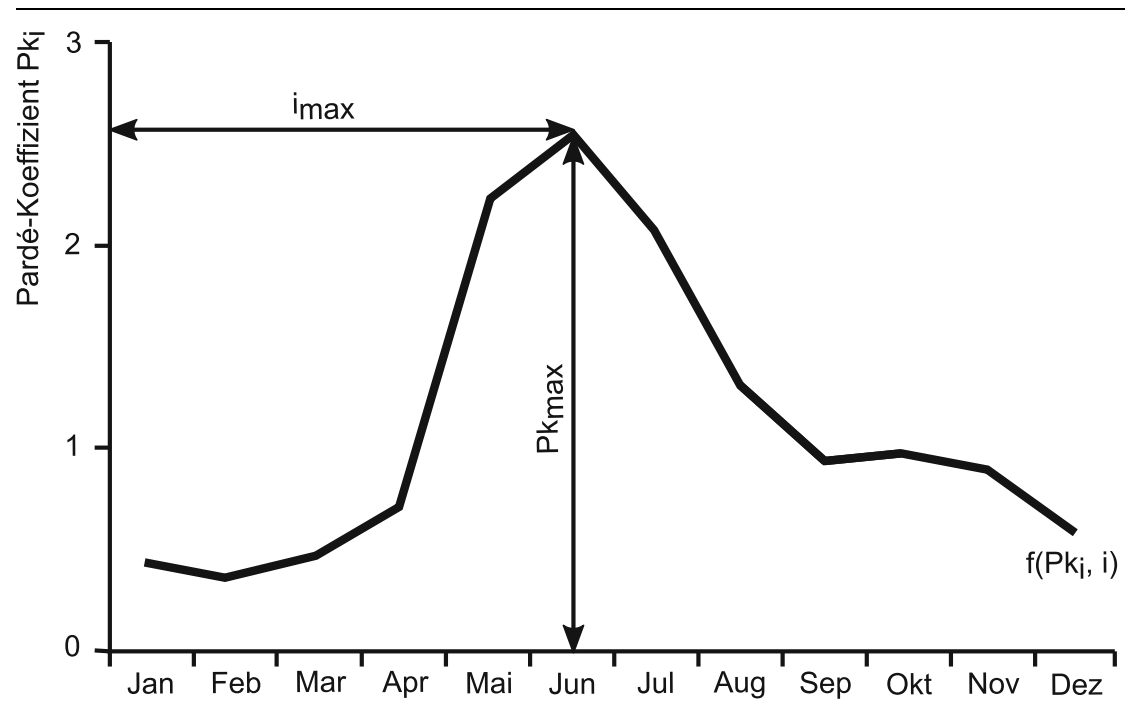

Monat i

Abb. 2 Beschreibung der Parameter zur Untersuchung der Saisonalität anhand eines nivalen Abflussregimes (nach Merz und Blöschl (2005), modifiziert)

Langfristig wird durch die Veränderung saisonaler Abflusscharakteristiken eine Verschiebung der Abflussregime in alpinen Gegenden erwartet.

Der Alpenraum Österreichs machte in den erwähnten überregionalen Studien der Regimeforschung meist nur einen geringen Anteil aus. Obwohl aus überregionaler Sicht wertvolle Informationen ermittelt werden konnten, wurden bei der Untersuchung oft nur ausgewählte Stationen berücksichtigt. Es ist uns keine österreichweite Untersuchung von Mittelwasserregimen bekannt, die basierend auf langjährigen Zeitreihen aller verfügbaren Messstellen die Abflussentwicklung untersucht und auf regionale Besonderheiten eingeht.

Das Ziel dieser Arbeit ist die Untersuchung anthropogen unbeeinflusster Mittelwasserregime in Österreich mit einem besonderen Schwerpunkt auf den Alpenraum. Im 50 Jahre umfassenden Bezugszeitraum werden Veränderungen im charakteristischen mittleren Wasserdargebot identifiziert. Diese werden anschließend ihrer zeitlichen sowie räumlichen Entwicklung gegenübergestellt und im Kontext der Trends der hydrologischen Größen analysiert. Es wird gezeigt, welche Abflussregimetypen vorkommen, wie sie sich verändert haben und wie vergangene und prognostizierte Veränderungen zueinander stehen.

\section{Daten und Methodik}

\subsection{Datengrundlage}

Für die Analyse der Mittelwasserregime im Bezugszeitraum von 1961 bis 2010 (50 Jahre) wurden die mittleren Monatsabflüsse an 280 Pegeln in Österreich untersucht. Die Daten wurden vom Hydrographischen Dienst Österreichs zur Verfügung gestellt. Für die Untersuchung wurden die Zeitreihen der mittleren monatlichen Abflüsse an Pegeln anthropogen unbeeinflusster Fließgewässer mit homogenen und konsistenten Abflussreihen herangezogen, deren Einzugsgebiete eine Mindestgröße von $10 \mathrm{~km}^{2}$ haben und keine Beeinflussung durch größere Seen aufweisen. Die Beschränkung der Daten auf monatliche Mittelwerte erfolgte, weil Mittelwasserregime meist anhand des Jahresgangs der Pardé-Koeffizienten (s.unten) beurteilt werden und diese aus Monatsmittelwerten berechnet werden. Abb. 1 zeigt die Verteilung der Pegel in Abhängigkeit von ihrer Höhenlage und Einzugsgebietsgröße.

\subsection{Untersuchungsgebiet}

Nach der Alpenkonvention (Ständiges Sekretariat der Alpenkonvention 2016) hat Österreich mit $28,7 \%$ den größten Anteil an der Gesamtfläche der Alpenregion, der wiederum $65 \%$ der gesamten Landesfläche ausmacht (Abb. 1). Ein wesentliches Merkmal von Gebirgszügen wie den Alpen ist die
Barrierewirkung, welche die großräumigen Windsysteme beeinflusst und eine orografische Hebung der Luftmassen bewirkt, die zur Niederschlagsbildung führt. Für die räumliche Verteilung der Niederschläge im Alpenraum selbst sind die Unterschiede zwischen Anströmseite (Luv) und Windschatten (Lee) entscheidend. Die Höhenabhängigkeit, ausgedrückt durch den Niederschlagsgradienten, ist zwar eine Grundcharakteristik des alpinen Niederschlags, sie variiert aber je nach Region, den topografischen Verhältnissen und der zeitlichen Auflösung der Niederschlagsdaten. Die größten Werte mit bis $\mathrm{zu} 100 \mathrm{~mm} / 100 \mathrm{~m}$ treten am Nordrand der Alpen auf, während in inneralpinen Bereichen deutlich kleinere oder sogar negative Gradienten auftreten können. Die mittlere Lufttemperatur nimmt mit zunehmender Höhe ab. Dies bestimmt die Schneefallgrenze und somit die Niederschlagsart, deren Speicherung und den Abflusszeitpunkt. Höhere Lagen sind stark von Schnee und Schneeschmelze beeinflusst, hingegen weisen niedrigere Lagen höhere Verdunstungsmengen auf. Die mittlere potenzielle Jahresverdunstung beträgt ca. $650 \mathrm{~mm}$ im Osten und $100 \mathrm{~mm}$ im Westen, der mittlere jährliche Gebietsniederschlag je nach Lage zwischen $480 \mathrm{~mm}$ im östlichen Flachland und $2700 \mathrm{~mm}$ in den Alpen im Westen (BMLFUW 2005). Durch die komplexe räumliche Heterogenität und ihre regionalen Besonderheiten variiert auch das Abflussgeschehen. Veränderungen haben nicht nur einen lokalen Einfluss, sondern sind auch für die Unterlieger von Bedeutung, da fast das gesamte Bundesgebiet Teil des Donaueinzugsgebietes ist, dessen Abfluss in das Schwarze Meer mündet.

\subsection{Analyse der Mittelwasserregime}

Die Saisonalität und Entwicklung langjähriger Mittelwasserabflüsse wurde anhand von Mittelwasserregimen untersucht. Der Zeitraum von 1961-2010 wurde durch die Betrachtung eines 10-Jahres-Fensters analysiert, welches von Beginn bis zum Ende der Periode in 1-Jahresschritten über den Zeitraum verschoben wurde (1961-1970, 1962-1971 usw. bis 2001-2010). Extremhochwässer, wie z. B. jenes im August 2002, wirken sich selbst im 10Jahresmittel deutlich aus. Um einer Verzerrung der Abflussregime durch Extremhochwässer vorzubeugen, wur- 


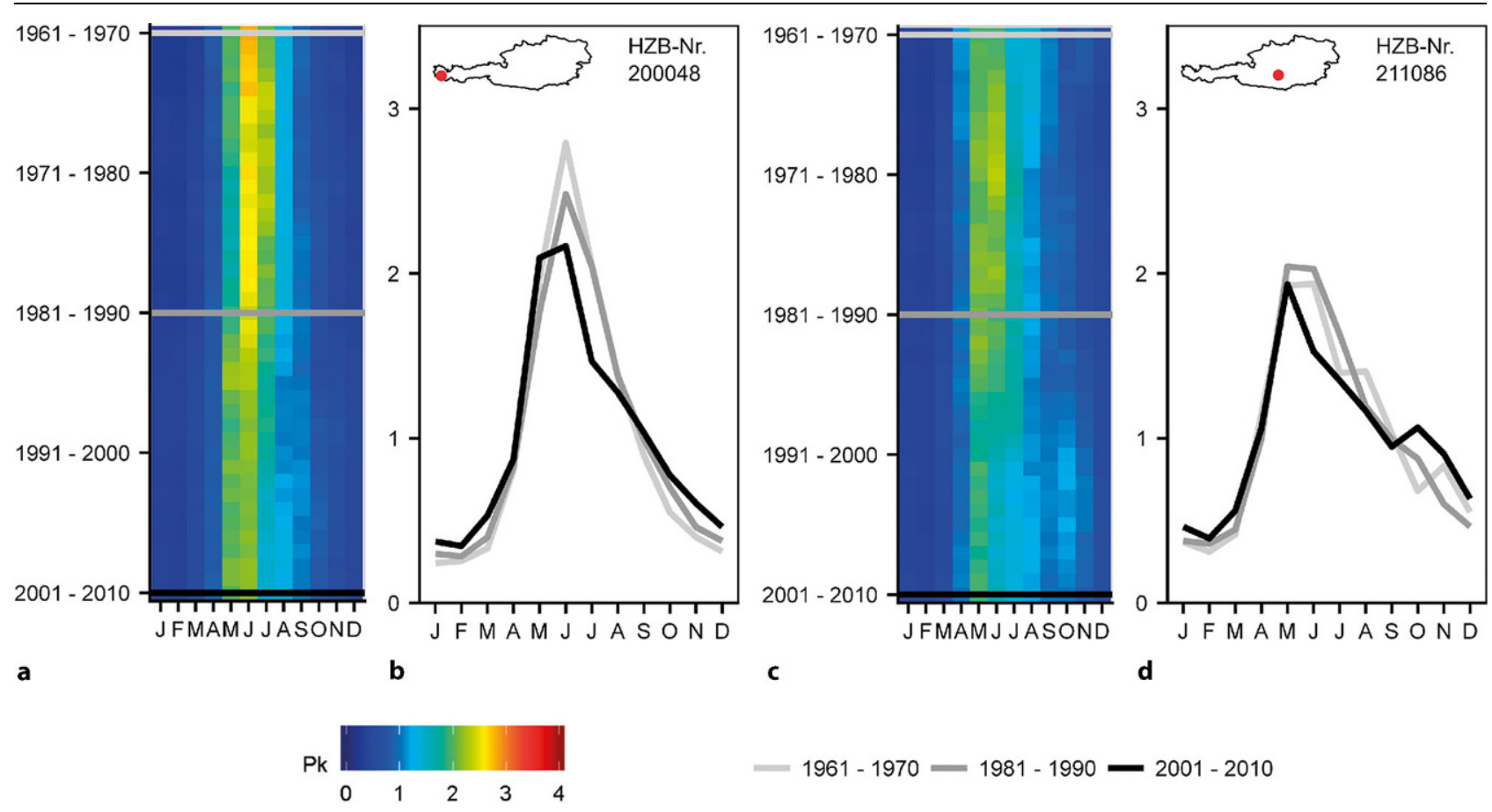

Abb. 3 Entwicklung nivalerAbflussregimeandenStationen200048-Schruns(Vonbunweg)anderLitz (a, b) und211086-Gestüthof an der Mur (c, d) basierend auf mittleren Pardé-Koeffizienten (Pk) untersuchter 10-Jahresreihen im Zeitraum 1961-2010

de bei der Berechnung des gleitenden Mittels je Zeitfenster das Jahr mit dem größten mittleren Monatsabfluss ausgeschieden.

Der mittlere Jahresgang des Abflusses je Zeitfenster wurde mittels PardéKoeffizienten Pk (Pardé 1933) beschrieben. Der $\mathrm{Pk}_{\mathrm{i}}$ ist definiert als Quotient aus dem mittleren monatlichen Abfluss und dem mittleren jährlichen Abfluss, für den gilt

$$
P k_{i}=\frac{12}{n} \sum_{j=1}^{n} \frac{Q_{i j}}{\sum_{i=1}^{12} Q_{i j}}
$$

mit Q als mittlerer Abfluss je Monat $\mathrm{i}$ und Jahr $\mathrm{j}$ bezogen auf die Länge der Zeitreihe $n$. Nach Pardé (1933) lässt sich aus der Ganglinie des mittleren Pk auf die dominierenden Abflussanteile schließen, also ob die Abflüsse glazialen, nivalen oder pluvialen Ursprungs sind.

Unter dem maximalen Pardé-Koeffizienten $\mathrm{Pk}_{\max }$

$$
P k_{\max }=\max \left(P k_{i}\right)
$$

versteht man den größten $\mathrm{Pk}$ im betrachteten Zeitfenster, der im Monat $\mathrm{i}_{\max }$ auftritt. Im Falle eines konstanten Abflusses ist der $\mathrm{Pk}_{\max }$ gleich eins. Hingegen bedeutet ein $\mathrm{Pk}_{\max }$ von 12 , dass der gesamte Jahresabfluss im be- trachteten Monat $\mathrm{i}_{\max }$ auftritt (Merz und Blöschl 2005). Indem die mittleren Monatsabflüsse über den mittleren Jahresabfluss normiert werden, kann die Vergleichbarkeit der mittleren $\mathrm{Ab}$ flüsse je 10-Jahres-Fenster eines Pegels und gleichzeitig auch überregional zwischen unterschiedlichen Pegeln hergestellt werden. Die Parameter $\mathrm{Pk}_{\max }$ und $\mathrm{i}_{\max }$ dienen in dieser Arbeit zur Beschreibung der Saisonalität und sind in Abb. 2 schematisch dargestellt.

Durch die Berechnung der $\mathrm{Pk}_{\mathrm{i}}$ für jeweils um ein Jahr verschobene 10-Jahres-Zeitfenster wurde der Einfluss der Abflussschwankungen von Jahr zu Jahr ausgeschlossen. Auf diese Weise wurden die charakteristischen saisonalen Abflussverläufe untersucht und Veränderungen identifiziert. Ihre Ausprägung wurde anhand des Auftretens von Maxima, ihrer Auftrittszeitpunkte und Größe bestimmt.

\subsection{Klassifikation von Regimen mittels Clusteranalyse}

Auf der Grundlage der nach Pardé quantifizierten Parameter wurde eine Clusteranalyse mittels k-Means-Algorithmus (Hartigan und Wong 1979) durchgeführt. Zunächst wurden für die Periode 1981-2010 Cluster mit Ähnlichkeiten im saisonalen Abfluss identifi- ziert. Als Anzahl der Cluster wurde fünf gewählt, da einerseits mit einer größeren Zahl die Abweichungsquadratsumme nicht mehr wesentlich verringert wurde, und andererseits mit fünf Clustern die wesentlichen Unterschiede zwischen den Stationen identifizierbar waren. Anhand derselben Distanzmaße wurden zum Vergleich die Beobachtungspunkte der Periode 1961-1990 den am nächsten liegenden Klassenschwerpunkten der bereits bestimmten Cluster zugeordnet.

\subsection{Darstellung des zeitlichen Verlaufs und der Saisonalität}

Für die Darstellung des saisonalen Abflussverhaltens wurden die mittleren Pk für jeden Monat und jedes der betrachteten Zeitfenster berechnet. Die erhaltene Matrix der $\mathrm{Pk}_{\mathrm{i}, \mathrm{j}}(\mathrm{i}=1 \ldots 12$, $\mathrm{j}=1 \ldots$ Anzahl der Zeitfenster) wurde als Bild dargestellt. Der farbliche Verlauf in horizontaler Richtung (in einer Zeile) zeigt den Jahresgang der $\mathrm{Pk}$ im jeweils betrachteten Zeitfenster. In vertikaler Richtung ist die zeitliche Entwicklung der Pk je Monat abgebildet. Der Farbton entspricht dabei jeweils der Größe der Pk.

Diese Darstellung wurde durch eine zweite ergänzt, die den Jahresgang der $\mathrm{Pk}$ für den ersten, mittleren und 


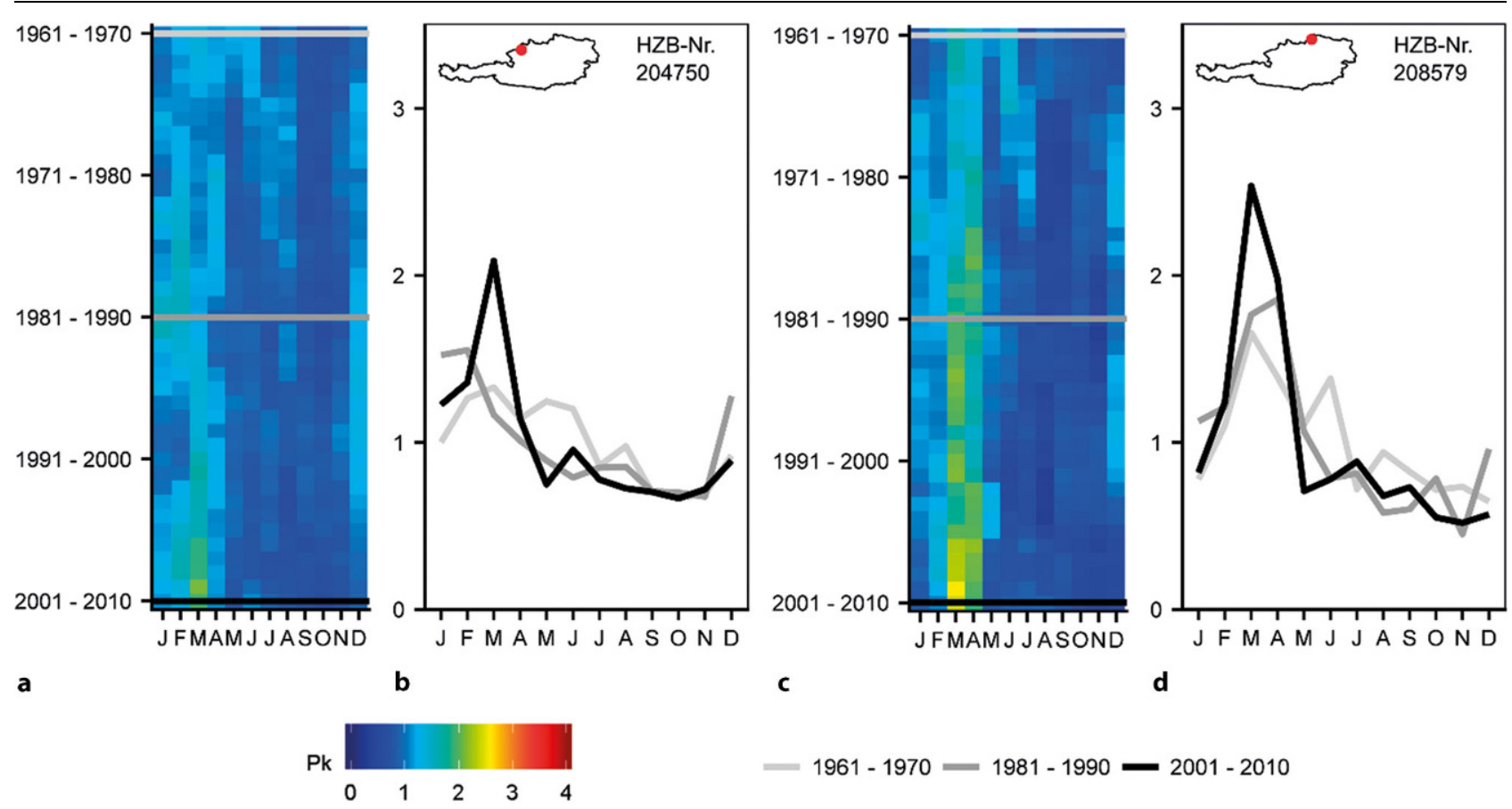

Abb. 4 Entwicklung pluvialer Abflussregime an den Stationen 204750-Haging an der Antiesen (a, b) und 208579-Hoheneich am Braunaubach (c, d) basierend auf mittleren Pardé-Koeffizienten (Pk) untersuchter 10-Jahresreihen im Zeitraum 1961-2010

letzten 10-Jahresabschnitt darstellt. Die Lage der ausgewählten Perioden wurde in der Bilddarstellung anhand von horizontalen Linien hervorgehoben.

\subsection{Räumliche Analyse und Vergleich mit hydrologischen Größen}

Die Ergebnisse der Regime- und Clusteranalyse wurden in Hinblick auf ihre räumliche Verteilung kartografisch aufbereitet. Veränderungen in der $\mathrm{Ab}$ flusscharakteristik wurden mit saisonalen Trends im Niederschlag, Abfluss und der Wasserbilanz im Hydrologischen Atlas Österreichs (HAÖ) verglichen (Fürst et al. 2005a, 2005b, 2005c).

\section{Ergebnisse}

\subsection{Beschreibung der Abflussregime}

Im Alpenraum sind die Abflussregime deutlich durch Akkumulation und Abbau der Schneedecke geprägt. Niederschlag, der als Schnee fällt, wird gespeichert und ist daher nicht direkt, sondern erst zeitversetzt bei der Schneeschmelze abflusswirksam. Bei Gletschern wird nicht nur eine saisonale Speicherung wirksam, sondern eine Speicherung und verzögerte Schmelzwasserabgabe über sehr lange Zeiträume von Jahrzehnten. In Einzugsge- bieten mit hohem Gletscheranteil tritt das Maximum der mittleren Monatsabflüsse im Jahresverlauf zur Zeit der Eisschmelze auf. Glaziale Abflussregime sind durch besonders hohe $\mathrm{Pk}$ in den abflussreichsten Sommermonaten und besonders niedrige $\mathrm{Pk}$ in den Wintermonaten charakterisiert. Mit abnehmendem Gletschereinfluss im Einzugsgebiet treten nivale Abflussregime auf. Der Jahresgang der mittleren monatlichen Abflüsse ist durch ein deutliches Abflussmaximum im Frühjahr gekennzeichnet. Mit zunehmendem Niederschlag als Regen nimmt die Spannweite zwischen maximalen und minimalen $\mathrm{Pk}$ ab. Rein pluviale Abflussregime sind durch einen ausgeglichenen Jahresgang der Pk erkennbar. Häufiger treten jedoch pluvio-nivale Mischformen auf, die durch Superposition pluvialer und nivaler Merkmale gekennzeichnet sind. Auf die räumliche Verteilung der Abflussregime wird in 3.3. Räumliche Analyse näher eingegangen. Nachfolgend werden Beispiele aufgetretener Abflussregime anhand repräsentativer Messstellen beschrieben. Die Kriterien für die Auswahl waren, dass Messdaten ab 1961 durchgehend vorhanden sind, der Verlauf typisch für das jeweilige Regime ist und die Pegel über das Bundesgebiet verteilt sind.
Abb. 3 zeigt die zeitliche Veränderung der nivalen Abflussregime an den Stationen Schruns (Vonbunweg) an der Litz in Vorarlberg (HZB-Nr. 200048) und Gestüthof an der Mur in der Weststeiermark (HZB-Nr. 211086). Deutlich zu erkennen ist die signifikante Dämpfung der Abflussspitze, die teils auch mit einer Verlagerung des abflussreichsten Monats in den Vormonat einhergeht. Die Teilabbildungen a und c lassen durch den vertikalen farblichen Verlauf der Pk in den abflussreichsten Monaten die Entwicklung der Dämpfung der Abflussspitze nachvollziehen. Typisch ist auch die Hebung der Herbst- bzw. Winterabflüsse, die ebenso durch den vertikalen Farbverlauf der $\mathrm{Pk}$ gezeigt wird. Der Jahresgang der Pk ist durch den horizontalen Farbverlauf jeder 10Jahresreihe ersichtlich und wurde für drei sich nicht überlappende Perioden (1961-1970, 1981-1990 und 2001-2010) in den Teilabbildungen b bzw. d dargestellt.

In Abb. 4 sind die Stationen Haging an der Antiesen in Oberösterreich (HZB-Nr. 204750) und Hoheneich am Braunaubach in Niederösterreich (HZB-Nr. 208579) dargestellt. Beide Stationen befinden sich nördlich der Alpen und zeigen im Gegensatz zu den vorherigen Beispielen Abflussregime mit überwiegend pluvialem Einfluss. 

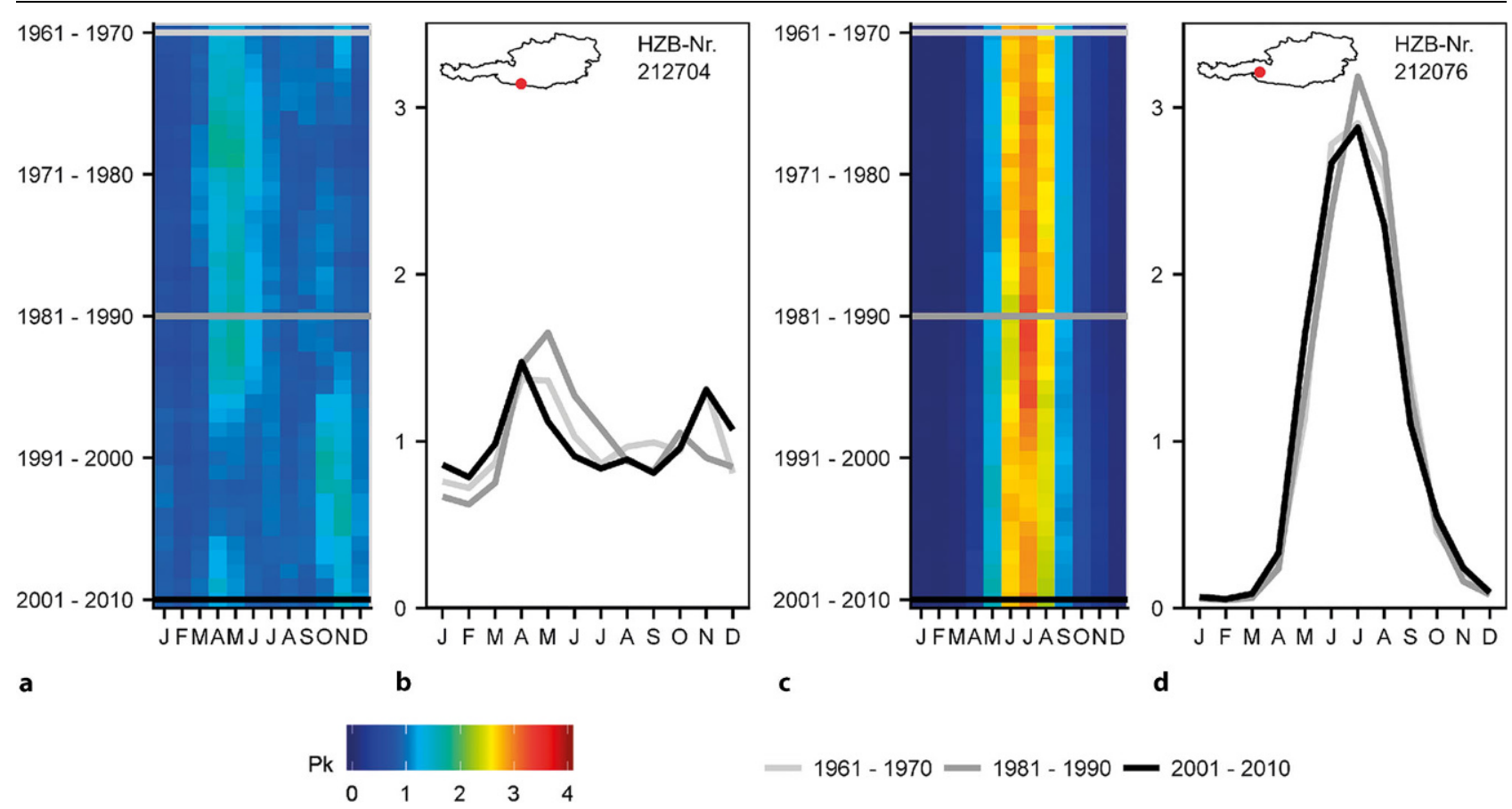

Abb. 5 Entwicklung pluvio-nivaler Abflussregime (Station 212704-Neudorf am Gösseringbach, a, b) und glazialer Abflussregime (Station 212076 - Matreier Tauernhaus am Tauernbach, c, d) basierend auf mittleren Pardé-Koeffizienten (Pk) untersuchter 10-Jahresreihen im Zeitraum 1961-2010

Die Abflusszunahme im Frühjahr ist in beiden Stationen zu erkennen und charakteristisch für Gegenden nördlich der Alpen. Nach den hohen Frühjahrsabflüssen ist der Verlauf der Pk ausgeglichen, wenn auch mit Schwankungen verbunden. Besonders an der Station Hoheneich ist die Zunahme der Frühjahrsabflüsse und die Abnahme der Sommer- und Herbstabflüsse in den letzten 50 Jahren deutlich zu erkennen.

In Abb. 5a und b ist am Beispiel der Station Neudorf am Gösseringbach im südlichen Kärnten (HZB-Nr. 212704) die für dieses Gebiet typische kontinuierliche Verlagerung des Abflussschwerpunktes vom Frühjahr in den Herbst erkennbar. An einigen Pegeln deuten die letzteren Pk-Verläufe eine mögliche Umkehr der Verschiebung an. Die Ursachen der Verlagerung sind in 3.4. Vergleich mit dem Trend der hydrologischen Größen beschrieben.

Abb. $5 c$ und $d$ zeigen ein glaziales Abflussregime an der Station Matreier Tauernhaus am Tauernbach im Norden Osttirols (HZB-Nr. 212076). Bei glazialen Regimen kam es teilweise um $1980 \mathrm{zu}$ einer Zunahme des maximalen mittleren Abflusses im Jahresgang, die in den nachfolgenden Jahren wieder abgenommen hat. In der zweiten Hälf- te des Frühjahrs haben die Abflüsse marginal, jedoch stetig zugenommen.

\subsection{Regimeklassifikation mittels Clusteranalyse}

Durch die Klassifikation mittels Clusteranalyse konnten für den Zeitraum 1981-2010 fünf Regionen bestimmt werden, die in weiterer Folge auch eine Abgrenzung räumlich homogener Regionen erlaubten (siehe 3.3. Räumliche Analyse). Im Bezugszeitraum 1961-1990 wurden die Abflussverteilungen je Pegel denselben Regionen zugeordnet. Die Abflussverteilungen jeder Station in einer Region sowie ihr jeweils mittlerer Verlauf (das Cluster-Zentrum) sind in Abb. 6 dargestellt.

Der Jahresgang der Pardé-Koeffizienten in Region 1 zeigt einen relativ ausgeglichenen Verlauf, mit $\mathrm{Pk}_{\max }=1,49$ und $\mathrm{Pk}_{\min }=0,71$. Es handelt sich dabei um die im Frühjahr (März, April) pluvial dominierten Regime im Flach- und Hügelland des Nordens und Ostens.

Die Region 2 ist ebenfalls Frühjahrspluvial geprägt, aber mit größerer Amplitude als Region $1\left(\mathrm{Pk}_{\max }=1,86\right.$ und $\left.\mathrm{Pk}_{\min }=0,62\right)$. Das Maximum der Abflüsse liegt eindeutig im März. Die Lage ist ebenfalls vorwiegend im Flach- und Hügelland des Nordens und Ostens, allerdings eher in größerer Höhenlage als Region 1.

Die Abflüsse in Region 3 sind deutlich nival beeinflusst, aber mit geringer Amplitude $\left(\mathrm{Pk}_{\max }=1,41\right.$ und $\left.\mathrm{Pk}_{\min }=0,62\right)$, wobei die Maxima im Mai, teilweise auch im April, auftreten. Während das Cluster-Zentrum eindeutig ein einfaches, unimodales Regime beschreibt, haben einige der zugehörigen Messstellen ein zwar geringes, aber doch eindeutiges Sekundärmaximum im Oktober oder November, welches pluvial bedingt ist. Diese Regime finden sich in den südlichen Ostalpen, dem Randgebirge östlich der Mur und vereinzelt auch in den nördlichen Ostalpen.

In Region 4 finden sich im Allgemeinen deutlich ausgeprägte, einfache Regime mit dem Maximum im Mai oder Juni $\left(\mathrm{Pk}_{\max }=2,08\right.$ und $\left.\mathrm{Pk}_{\min }=0,36\right)$. Es handelt sich um die nivalen Regime der zentralen und nördlichen Ostalpen. Bei vier Stationen gibt es allerdings gering ausgeprägte sekundäre Maxima im Oktober.

Region 5 schließlich enthält die glazialen Regime, die insgesamt die größte Amplitude der Pardé-Koeffizienten aufweisen $\left(\mathrm{Pk}_{\max }=2,85\right.$ und $\left.\mathrm{Pk}_{\min }=0,11\right)$. Auf die stark ausgeprägte Abflussspitze im Sommer, meist im Juli, folgt eine 

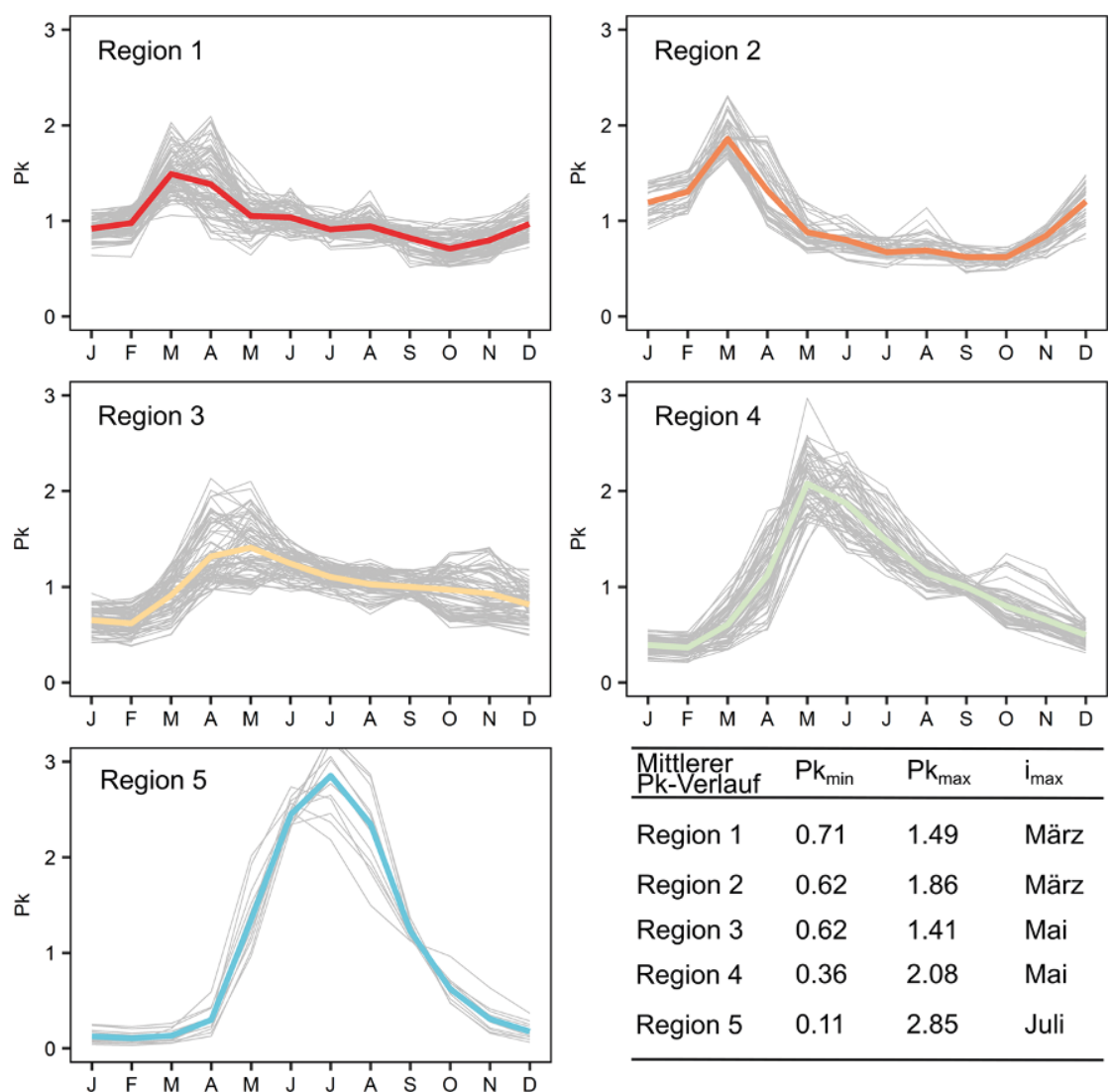

\begin{tabular}{|c|c|c|c|}
\hline $\mathrm{J} F \mathrm{M}$ & $\mathrm{M} \mathrm{J}$ & $\mathrm{J} A \mathrm{~S}$ & $O N D$ \\
\hline $\begin{array}{l}\text { Mittlerer } \\
\text { Pk-Verlauf } \\
\end{array}$ & $\mathrm{Pk}_{\text {min }}$ & $\mathrm{Pk}_{\max }$ & $\mathrm{i}_{\max }$ \\
\hline Region 1 & 0.71 & 1.49 & März \\
\hline Region 2 & 0.62 & 1.86 & März \\
\hline Region 3 & 0.62 & 1.41 & Mai \\
\hline Region 4 & 0.36 & 2.08 & Mai \\
\hline Region 5 & 0.11 & 2.85 & Juli \\
\hline
\end{tabular}

Abb. 6 Abflussregime der Clusteranalyse mit Verläufen der Pardé-Koeffizienten $(P k)$ der zugeordneten Stationen ( $g r a u$ ) und dem mittleren Pk-Verlauf je Region ( $f e t t)$. Der kleinste und größte Pardè-Koeffizient $\left(\mathrm{Pk}_{\min }, \mathrm{Pk}_{\max }\right)$ sowie der Monat $\mathrm{i}_{\max }$, im dem $\mathrm{Pk}_{\max }$ auftritt, sind für den mittleren Pk-Verlauf je Region in der Tabelle zusammengefasst

längere Phase mit geringen Abflüssen zwischen Spätherbst und der zweiten Hälfte des Frühjahrs.

Die Regionen 1 und 2 haben ihr Abflussminimum im Herbst, meist im Oktober, die Regionen 3 bis 5 im Winter, meist im Februar.

\subsection{Räumliche Analyse}

Die Prägung des österreichischen Bundesgebiets durch die Alpen spiegelt sich auch in der räumlichen Verteilung der Mittelwasserregime wider, die ein sehr klares räumliches Muster aufweist. Eine detaillierte Darstellung der zeitlichen Entwicklung saisonaler Abflusscharakteristiken und ihrer räumlichen Verteilung mit den Regimen für alle Stationen wird als Online-Zusatzmaterial zur Verfügung gestellt (Onlinematerial 1). Abb. 7 zeigt einen repräsentativen Ausschnitt daraus, der einen Nord-SüdSchnitt in der Umgebung der Venedigergruppe darstellt. Basierend auf der Untersuchung der 10-Jahreszeitreihen im gesamten Bundesgebiet treten in
Nord-Süd-Richtung folgende Regimetypen auf: Das Mühl- und Waldviertel sind charakterisiert durch Abflussregime mit pluvial dominiertem Verlauf der Pk. Im nördlichen Alpenvorland nehmen mit zunehmender Höhe nivale Merkmale zu. In der Alpenregion nördlich des Alpenhauptkamms treten nival geprägte Abflussregime auf. Den Einflussbereich vergletscherter Regionen bestimmen Abflussregime mit glazial geprägter Abflusscharakteristik. Südlich des Alpenhauptkamms treten pluvio-nivale Abflussregime auf, deren abflussstärkstes Monat nival verursacht im Frühjahr liegt, die aber auch im Oktober und November ein - meist schwächer ausgeprägtes - pluviales Sekundärmaximum aufweisen können. An einigen Stationen liegt das primäre Maximum durchaus während einiger 10-Jahresperioden im Herbst.

Auch in der Verteilung der fünf Regionen, die durch die Clusteranalyse im Bezugszeitraum von 30 Jahren bestimmt wurden, zeigte sich deutlich ein räumliches Muster (Abb. 8), das stark von der Höhenlage geprägt ist. Die von glazio-nivalen Einflüssen bestimmte, unimodal verteilte Region 5 trat ausschließlich in den hochalpinen Regionen der Venedigergruppe, Ötztaler und Zillertaler Alpen sowie dem Dachsteingebirge auf. Die nival geprägte Region 4 war in weiten Teilen des Gebietes der zentralen und nördlichen Ostalpen in Österreich vertreten. In den südlichen Ostalpen, dem Randgebirge östlich der Mur und vereinzelt auch in den nördlichen Ostalpen trat das Abflussregime der Region 3 auf. Im Flachund Hügelland im Norden und Osten (inkl. Böhmische Masse) dominierte die mittlere monatliche Abflussverteilung der pluvial geprägten Regionen 1 und 2.

Der Vergleich der Clusteranalysen für die betrachteten Perioden 1961-1990 und 1981-2010 zeigte, dass es zwischen diesen beiden Perioden sehr wohl Regimeveränderungen gegeben hat. Diese traten im Übergangsbereich des Alpenraumes bzw. seinen Rändern und vereinzelt im nördlichen Flach- und Hügelland auf. Durch die Verlagerung des abflussreichsten Monats von der ersten auf die zweite Hälfte des Frühjahrs entwickelte sich im südlichen Burgenland das Abflussregime der Region 1 bei einigen Pegeln zum Regime der Region 3. In Südkärnten sowie nördlich des Alpenhauptkamms kam es durch die Abnahme des nivalen Einflusses mitunter zur Verschiebung des Abflussregimes der Region 4 in die Region 3. Die zunehmende pluviale Ausprägung veränderte das Abflussregime des nördlichen Alpenvorlandes, das in der ersten Periode der dritten und in der zweiten Periode der ersten Region zugeordnet wurde. Eine Zunahme der Frühjahrsabflüsse führte im Flach- und Hügelland nördlich der Alpen vereinzelt zur Verschiebung des Regimes der Region 1 in die Region 2. Die Abflussregime in Vorarlberg und den Zentralalpen waren bis auf wenige Ausnahmen nicht von Regimeverschiebungen zwischen diesen beiden Perioden betroffen.

Die räumliche Verteilung der Abflussregime je Region und betrachteter Periode ist in der Abb. 8 kartografisch dargestellt.

\subsection{Vergleich mit dem Trend der hydrologischen Größen}

Die Analysen der Abflussregime lassen keinen direkten Schluss auf einen Trend der Abflüsse selbst, sondern nur auf deren jahreszeitliche Verteilung zu 


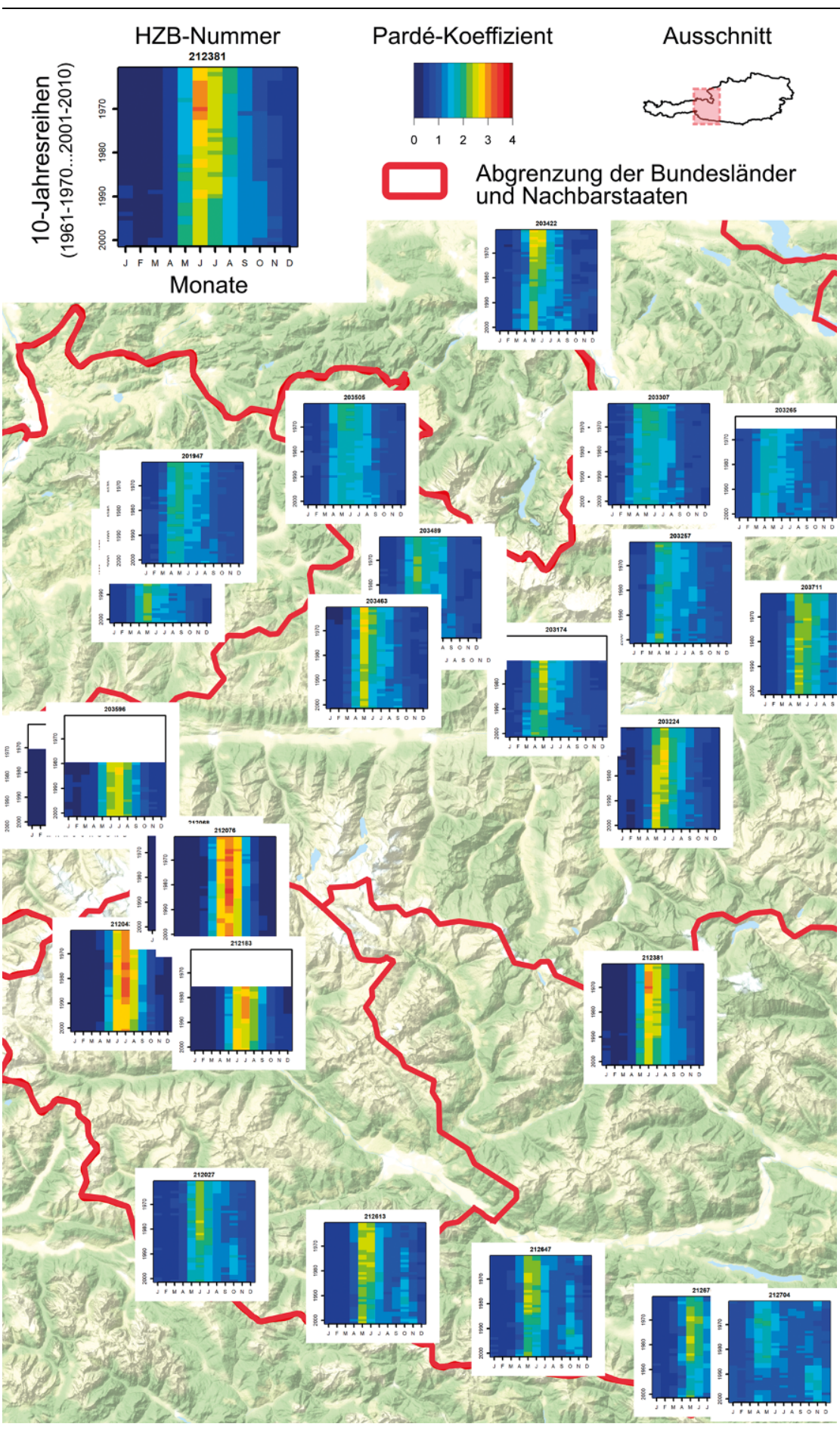

Abb. 7 Zeitliche Entwicklung saisonaler Abflusscharakteristiken und ihrer räumlichen Verteilung (repräsentativer Kartenausschnitt; für die Darstellung aller Stationen siehe Onlinematerial 1)

(der Mittelwert eines Jahresverlaufs von Pardé-Koefflizienten ist per definitionem immer 1). Im folgenden $\mathrm{Ab}$ schnitt wird daher diskutiert, wie weit die Veränderungen der Regime mit den im Hydrologischen Atlas Österreichs (HAÖ) publizierten saisonalen Trends lich des Alpenhauptkammes starken negativen Trends des Abflusses im Sommerquartal (Juni/Juli/August) ähnlich große positive Trends im Winter- und Frühjahrsquartal (Dezember/Jänner/ Februar bzw. März/April/Mai) gegenüberstehen. Südlich des Alpenhauptkammes sind die Trends im Abfluss in allen Jahreszeiten mehr oder weniger signifikant negativ, im Frühjahr und Sommer fast überall deutlich.

Die Abflussregime an den nördlich des Alpenhauptkamms gelegenen Pegeln zeigten eine mit den Trendanalysen konsistente Reaktion auf die Veränderungen der hydrologischen Größen. Insbesondere im Bereich der Voralpen und an der Nordabdachung der Alpen werden die Regime tendenziell ausgeglichener, das heißt, die Abflussspitzen im April und Mai bis Juni werden kleiner, während die bisherigen Niederwasserphasen im Herbst und Winter größere Abflussanteile bekommen. Teilweise ist auch eine früher auftretende Abflussspitze festzustellen. Wegen der Verwendung von Monatsmitteln des Abflusses ist die zeitliche Auflösung entsprechend eingeschränkt und dieser Effekt daher nur bei wenigen Messstellen erkennbar.

Im Gebiet südlich des Alpenhauptkamms kam es zu einer Verschiebung von Abflussanteilen vom Frühjahr und Sommer in den Herbst bzw. Winter. Mit der Trendanalyse der Abflüsse ist diese Feststellung insofern konsistent, als die negativen Trends im Frühjahr und Sommer deutlich stärker waren als im Herbst und Winter.

\section{Diskussion und Conclusio}

\subsection{Zeitliche Entwicklung der \\ Abflussregime und ihre räumliche Verteilung}

Die Untersuchung der Abflussregime und ihrer zeitlichen Entwicklung im betrachteten Zeitraum von 1961-2010 zeigte, dass auch bei Verwendung von gleitenden 10-Jahresperioden von Periode zu Periode noch große Schwankungen auftreten. Dennoch sind auch kontinuierliche Veränderungen erkennbar, die als Trends in der Entwicklung interpretiert werden können. Es konnte gezeigt werden, dass Veränderungen in der saisonalen Abflusscharakteristik an anthropogen unbeeinflussten Fließgewässern nicht abrupt auftraten, sondern sich über einen längeren Zeitraum erstreckten. $\mathrm{Zu}$ den beob- 


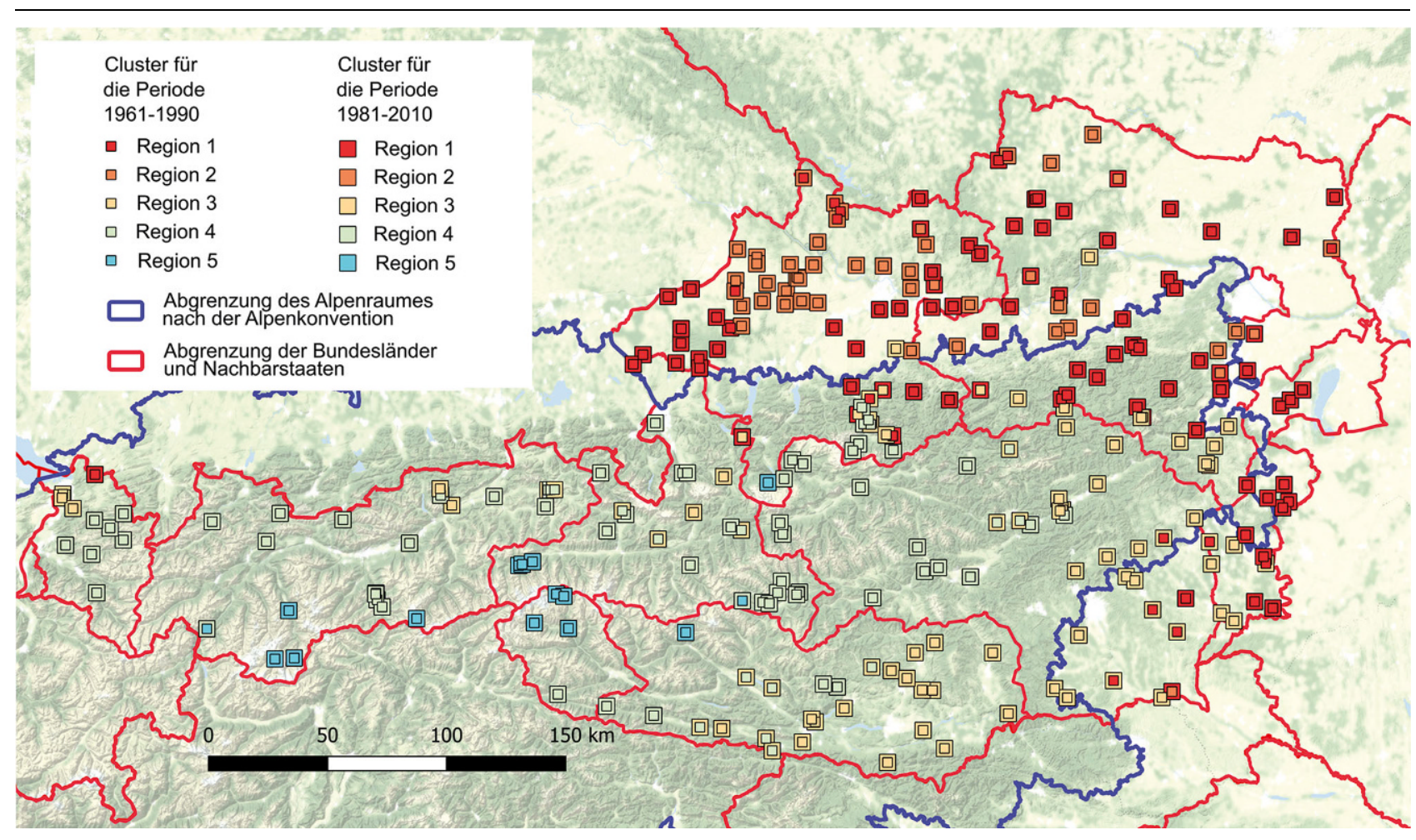

Abb. 8 Vergleich der klassifizierten Abflussregimegruppen der Perioden 1961-1990 (kleine Symbole) und 1981-2010 (große Symbole)

achteten Veränderungen gehörten die Verlagerung des Abflussschwerpunkts und somit eine Veränderung der Saisonalität, oder auch eine Veränderung der Größe des maximalen Abflusses im Jahresverlauf (Dämpfung, Erhöhung) inkl. Verschiebung seines Auftrittszeitpunkts. Es kam auch zur Anhebung der Abflüsse in abflussarmen Zeiten bis hin zur Ausbildung von zusätzlichen Maxima.

Der Auftrittsmonat und die Größe des maximalen Pardé-Koeffizienten im Jahresverlauf ( $\mathrm{i}_{\max }$ und $\mathrm{Pk}_{\max }$ ) sind vor allem bei einfachen (unimodalen) Regimen aussagekräftige Kenngrößen (siehe auch Merz und Blöschl 2005). Vorsicht ist geboten bei sehr ausgeglichenen und komplexen Regimen mit zwei oder mehreren ähnlich hohen Maxima. Hier können geringfügige Unterschiede die Rangfolge der Maxima verändern und fälschlicherweise eine Verschiebung der Saisonalität indizieren. Von dieser Gefahr sind auch Klassifikationen betroffen, die auf der Rangfolge von Maxima und ihrer Auftrittszeitpunkte beruhen, wie etwa die Klassifikationen nach Pardé (1933), Grimm (1968) oder Mader et al. (1996). Eine starre Fixierung einzelner Merkmale führt zu einem statischen Begriffsverständnis.
Da Abflussregime ein dynamisches System beschreiben, stünde eine starre Annahme dieses Begriffs zu diesem im Widerspruch (Krasovskaia et al. 1999). Daher wurde in dieser Arbeit die Klassifikation anhand einer Clusteranalyse mittels k-Means-Algorithmus durchgeführt, die auf einer Einteilung anhand unterschiedlicher unabhängiger Merkmale beruht. Die Ergebnisse wurden im Zusammenhang mit den hydrologischen Größen und der Veränderung der saisonalen Wasserbilanz betrachtet.

In der Clusteranalyse wurden fünf Regimeklassen bestimmt. Die Verteilung der Abflussregime ergab sich entsprechend der Höhenlage und den in diesem Zusammenhang dominierenden klimatischen Faktoren. Eine besondere Rolle nahm hierbei der Alpenhauptkamm als trennendes Element in Nord-Süd-Richtung ein. Der Vergleich zwischen den je 30 Jahre umfassenden Perioden 1961-1990 und 1981-2010 zeigte, dass in großen Teilen des Alpenraums sowie des Flachund Hügellandes mit dieser Art der Klassifikation keine systematischen Regimeveränderungen nachweisbar sind, da die monatliche Auflösung und die geringe Anzahl an Klassen kleinere Verschiebungen nicht erkennen lassen.
Nur im Übergangsbereich des Alpenraums bzw. seinen Rändern und im Flach-und Hügelland sind Regimeveränderungen auch in einer veränderten Klassenzuordnung erkennbar. Die gleitenden Abflussmittelwerte der 10Jahreszeitreihen wiesen an diesen wie auch anderen Pegeln unterschiedlich ausgeprägte, jedoch deutlich sichtbare Schwankungen auf. Das heißt auch, dass die Verwendung von 10-jährigen Zeitreihen noch keine stabile Regimeklassifikation zulässt.

Abflussregime werden durch unterschiedliche, meist höhenabhängige hydrologische Größen bestimmt. Die Reaktion der Regime ist folglich an Veränderungen ihrer bestimmenden Faktoren gebunden. Mit der Höhenlage verändert sich auch der Einfluss der Schneeverhältnisse (Akkumulation, Schmelze, Schneerücklage). Insofern traten in alpinen Gegenden die höchsten mittleren Monatsabflüsse in den Sommermonaten auf (Schmelzvorgänge). Mit abnehmender Höhenlage trat der abflussreichste Monat früher auf und war im Tiefland bereits in der ersten Hälfte des Frühjahres zu finden. 


\subsection{Der Trend im Vergleich und seine Folgen}

Die beobachteten Trends der untersuchten 50 Jahre zeigten eine deutliche Zunahme pluvialer Einflüsse auf regimebestimmende Merkmale. Allgemein kam es zu einer Zunahme der Frühjahrsabflüsse, die häufig mit reduzierten Sommerabflüssen auftrat. Diese Kombination führte zu einer Dämpfung des maximalen mittleren Pardé-Koeffizienten im Jahresverlauf und gegebenenfalls auch zu dessen Verschiebung in den Vormonat. Von diesen Veränderungen waren sowohl nivale als auch pluviale Abflussregime betroffen. Nivale Regime wiesen zusätzlich zunehmende Herbst- bzw. Winterabflüsse auf. Im Süden Österreichs konnte eine signifikante Reduktion der Sommerabflüsse festgestellt werden sowie eine Verschiebung des Abflussschwerpunktes vom Frühjahr in den Herbst.

Trotz des dramatischen Gletscherschwunds der letzten Jahrzehnte wiesen die saisonalen Abflusscharakteristiken vergletscherter Regionen durchgehend eindeutig glaziale Merkmale auf. Studien zur Untersuchung der Gletscher und Gletscherabflüsse prognostizieren für die nahe Zukunft zwar weiterhin eine Zuordnung zu glazialen Regimen, jedoch deuten sie bis 2100 auf eine Verschiebung hin zum nivalen Abflussregime mit einem früheren Abflussmaximum und Anhebung der Winterabflüsse (Hanzer et al. 2018; Förster et al. 2015; Weber et al. 2010). Diese signifikanten Veränderungen stehen im Zusammenhang mit einem sich erwärmenden Klima (Bard et al. 2015).

Die beobachteten Veränderungen von Abflussregimen in der zeitlichen Entwicklung entsprachen den Inhalten des HAÖ (BMLFUW 2005), insbesondere der Entwicklung der hydrologischen Größen (vgl. 3.4. Vergleich mit dem Trend der hydrologischen Größen). Die Ergebnisse dieser Arbeit sind auch im Kontext des Klimawandels besonders interessant, da im Alpenraum die mittlere Zunahme der Lufttemperatur stärker ausgeprägt ist als im globalen Mittel (Böhm 2008). Für Österreich wird zudem mit einer zunehmenden jährlichen Sonnenscheindauer in den Alpen, einem Anstieg der Schneefallgrenze sowie einer Zunahme der Niederschlagsmenge im Norden bzw. Westen und einer Abnahme im Süden zu rechnen sein (APCC 2014; Blöschl et al. 2017). Der APCC weist ebenso auf Auswirkungen auf den Wasserkreislauf hin, sodass mit einer Verschiebung der Saisonalität des Abflusses zu rechnen ist. Es wird eine fallende Tendenz der Sommerabflüsse erwartet, die vor allem im Süden deutlich ausgeprägt sein wird.

Der Anstieg der Schneefallgrenze verringert den Anteil von Schnee am Gesamtniederschlag in den betroffenen Gebieten und verändert somit das Wasserdargebot. Durch den Rückgang der Schneeakkumulation in alpinen Einzugsgebieten und der Zunahme von Regenniederschlägen werden die Winterabflüsse im Mittel zunehmen und zu einer Verschiebung der Niederwasserperiode in den Herbst bzw. Spätsommer führen (Zampieri et al. 2015; APCC 2014). Das hohe Wasserdargebot im Sommer bzw. Frühjahr wird weiter abnehmen und im Jahresverlauf früher auftreten (Frey et al. 2013). In Zukunft werden vor allem der März und April von Schmelzwasserabflüssen geprägt sein (Laghari et al. 2012).

Die Verschiebung der Niederwasserperiode von Winter in den Spätsommer bzw. Herbst führt in Zukunft zu einer Verringerung des Abflussanteils aus den Alpen, die sowohl lokale als auch flussabwärts gelegene Einzugsgebiete der Donau prägen wird (Vanham und Rauch 2009). Für die Energieerzeugung aus Wasserkraft bedeutet es, dass die Anzahl der Tage mit Niedrigwasser im Winter in derzeit nival geprägten Regionen abnehmen wird (Goler et al. 2016). Generell ist zukünftig jedoch mit einer verringerten Energieproduktion in Ös- terreich zu rechnen (Stanzel und Nachtnebel 2010). Vor allem eine Verlagerung des Wasserdargebots ist für wasserwirtschaftliche Belange (Koboltschnig und Schöner 2011; Blöschl et al. 2011; Holzmann und Koboltschnig 2010) wie auch für das Umweltmanagement (Schneider et al. 2013) gleichermaßen bedeutsam. Eine solche Verlagerung könnte mit negativen Folgen für die Wasserqualität in Niederwasserperioden verbunden sein (Nilsson und Renöfält 2008). Auch Auswirkungen auf Fließgewässerökosysteme, ihre Artenzusammensetzung, die Gewässertemperaturen und den ökologischen Gewässerzustand sind hier von großem Interesse (Brown et al. 2018; Laizé et al. 2014; Perkin und Bonner 2011; Bunn und Arthington 2002).

Der Vergleich zwischen den beobachteten Entwicklungen der Mittelwasserabflussregime und den prognostizierten Abflussveränderungen zeigt, dass die beobachteten Entwicklungen als sich bereits abzeichnende klimabedingte Abflussveränderungen im $\mathrm{Al}$ penraum gesehen werden können, die sich in der Zukunft weiter fortsetzen werden. Für die Hydrologie Österreichs sind sie bereits heute von großer Bedeutung.

Funding Open access funding provided by University of Natural Resources and Life Sciences Vienna (BOKU).

Open Access Dieser Artikel wird unter der Creative Commons Namensnennung 4.0 International Lizenz (http:// creativecommons.org/licenses/by/4. 0/deed.de) veröffentlicht, welche die Nutzung, Vervielfältigung, Bearbeitung, Verbreitung und Wiedergabe in jeglichem Medium und Format erlaubt, sofern Sie den/die ursprünglichen $\mathrm{Au}$ tor(en) und die Quelle ordnungsgemäß nennen, einen Link zur Creative Commons Lizenz beifügen und angeben, ob Änderungen vorgenommen wurden.
APCC (2014): Österreichischer Sachstandsbericht Klimawandel 2014 (AAR14). Austrian Pane on Climate Change (APCC), Verlag der Österreichischen Akademie der Wissenschaften, Wien Bard, A., Renard, B., Lang, M., Giuntoli, I., Korck, J, Koboltschnig, G., Janza, M., D'Amico, M., Volken, D. (2015): Trends in the hydrologic regime of Alpine rivers. Journal of Hydrology 529:1823-1837. doi:https://doi.org/10.1016/j. jhydrol.2015.07.052
Blöschl, G., Schöner, W., Kroiß, H., Blaschke, A. P., Böhm, R., Haslinger, K., Kreuzinger, N., Merz, R., Parajka, J., Salinas, J. L., Viglione, A. (2011): Anpassungsstrategien an den Klimawandel für Österreichs Wasserwirtschaft - Ziele und Schlussfolgerungen der Studie für Bund und Länder. Österreichische Wasser- und Abfallwirtschaft 63 (1-2):1-10. doi:https://doi.org/10. 1007/s00506-010-0274-2
Blöschl, G., Parajka, J., Blaschke, A. P., Hofstätter, M., Haslinger, K. und Schöner, W. (2017): Klimawandel in der Wasserwirtschaft - Schwerpunkt Hochwasser, Dürre und Trockenheit. Bundesministerium für Land- und Forstwirtschaft, Umwelt und Wasserwirtschaft, Wien, S 99 BMLFUW (Hrsg) (2005): Hydrologischer Atlas Österreichs. Bundesministerium für Land- und Forstwirtschaft, Umwelt und Wasserwirtschaft, Wien 
Böhm, R. (2008): Heiße Luft: Reizwort Klimawandel - Fakten, Ängste, Geschäfte. Edition Va Bene, Wien

Bower, D., Hannah, D. M., McGregor, G. R. (2004): Techniques for assessing the climatic sensitivity of river flow regimes. Hydrological Processes 18:2515-2543. doi:https://doi.org/10. 1002/hyp.1479

Brown, L. E., Khamis, K., Wilkes, M., Blaen, P., Brittain, J. E., Carrivick, J. L., Fell, S., Friberg, N., Füreder, L., Gislason, G. M., Hainie, S., Hannah, D. M., James, W. H. M., Lencioni, V., Olafsson, J. S., Robinson, C. T., Saltveit, S. J., Thompson, C., Milner, A. M. (2018): Functional diversity and community assembly of river invertebrates show globally consistent responses to decreasing glacier cover. Nature Ecology \& Evolution 2:325-333. doi:https://doi.org/10. 1038/s41559-017-0426- $\mathrm{x}$

Bunn, S. E., Arthington, A. H. (2002): Basic Principles and Ecological Consequences of Altered Flow Regimes for Aquatic Biodiversity. Environ mental Management 30 (4):492-507. doi:https:// doi.org/10.1007/s00267-002-2737-0

Förster, K., Oesterle, F., Hanzer, F., Huttenlau, M., Strasser, U. (2015): Bestimmung de Auswirkungen des Klimawandels auf die Gletscherdynamik und das Abflussregime im Rofental unter Verwendung eines gekoppelten glaziohydrologischen Modells. Ergebnisse aus dem Forschungsprojekt MUSICALS. In. Innsbrucker Jahresbericht 2014-2015. Innsbrucker Geographische Gesellschaft, Institut für Geographie der Universität Innsbruck, Innsbruck, S 240

Frey, S., Goler, R., Formayer, H., Holzmann, H. (2013): Die Auswirkung möglicher Klimawandelszenarien auf das Erzeugungspotenzia von Wasserkraftwerken. In. Weingartner, R. und Schädler, B. (Hrsg) Wasserressourcen im globalen Wandel, Hydrologische Grundlagen - von der Messung zur Anwendung, Forum für Hydrologie und Wasserbewirtschaftung, FgHW Schriftenreihe „Forum für Hydrologie und Wasserbewirtschaftung", Heft 32.13, S 145-152

Fürst, J., Kling, H., Nachtnebel, H. P. (2005a): Saisonale Trends in der Wasserbilanz. In: BMLFUW (Hrsg). Hydrologischer Atlas Österreichs. Bundesministerium für Land- und Forstwirtschaft, Umwelt und Wasserwirtschaft, Wien, S Karte 7.3

Fürst, J., Nachtnebel, H. P., Kling, H. (2005b): Saisonale Trends im Abfluss. In: BMLFUW (Hrsg). Hydrologischer Atlas Österreichs. Bundesministerium für Land- und Forstwirtschaft, Umwelt und Wasserwirtschaft, Wien, S Karte 5.10

Fürst, J., Nachtnebel, H. P., Kling, H. (2005c): Saisonale Trends im Niederschlag. In: BMLFUW (Hrsg). Hydrologischer Atlas Österreichs. Bundesministerium für Land- und Forstwirtschaft, Umwelt und Wasserwirtschaft, Wien, S Karte 2.1

Fürst, J., Kling, H., Nachtnebel, H. P., Hörhan, T. (2010): Trends in hydrologischen Variablen und in der Wasserbilanz Österreichs. In: ÖWAV (Hrsg). Auswirkungen des Klimawandels au Hydrologie und Wasserwirtschaft in Österreich. Präsentation der aktuellen Studien. Eigenverlag des Österreichischen Wasser- und Abfallwirtschaftsverbandes, Wien, S 202

Goler, R., Frey, S., Formayer, H., Holzmann, H. (2016): Influence of Climate Change on Rive Discharge in Austria. Meteorologische Zeitschrift 25 (5):621-626. doi:https://doi.org/10. $1127 /$ metz/2016/0562

Grimm, F. (1968): Das Abflussverhalten in Europa - Typen und regionale Gliederung. In Wissenschaftliche Veröffentlichungen des Deutschen Instituts für Länderkunde (Hrsg). Bd 25/26. VEB Bibliographisches Institut, Leipzig
Hall, J., Arheimer, B., Borga, M., Brázdil, R., Claps, P., Kiss, A., Kjeldsen, T. R., Kriauciuniene, J., Kundzewicz, Z. W., Lang, M., Llasat, M. C., Macdonald, N., McIntyre, N., Mediero, L., Merz, B., Merz, R., Molnar, P., Montanari, A., Neuhold, C. Parajka, J., Perdigao, R. A. P. Plavcová, L. Rogger, M., Salinas, J. L., Sauquet, E., Schär, C., Szolgay, J., Viglione, A., Blöschl, G. (2014): Understanding flood regime changes in Europe: a state-of-the-art assessment. Hydrology and Earth System Science 18:2735-2772. doi:https:// doi.org/10.5194/hess-18-2735-2014

Hanzer, F., Förster, K., Nemec, J., Strasser, U. (2018): Projected cryospheric and hydrological impacts of 21st century climate change in the Ötztal Alps (Austria) simulated using a physically based approach. Hydrology and Earth System Science 22:1593-1614. doi:https://doi.org/10. 5194/hess-22-1593-2018

Hartigan, J. A., Wong, M. A. (1979): A K-means clustering algorithm. Applied Statistics 28:100108

Holzmann, H., Koboltschnig, G. (2010): Hydrologische Veränderungen in hochalpinen Einzugsgebieten. In: ÖWAV (Hrsg). Auswirkungen des Klimawandels auf Hydrologie und Wasserwirtschaft in Österreich. Präsentation der aktuellen Studien. Eigenverlag des Österreichischen Wasser- und Abfallwirtschaftsverbandes, Wien, S 202

Jungwirth, M., Haidvogl, G., Moog, O., Muhar, S., Schmutz, S. (2003): Angewandte Fischökologie an Fließgewässern. Facultas Universitätsverlag, Wien

Koboltschnig, G., Schöner, W. (2011): The relevance of glacier melt in the water cycle of the Alps: the example of Austria. Hydrology and Earth System Science 15:2039-2048. doi:https:// doi.org/10.5194/hess-15-2039-2011

Krasovskaia, I., Gottschalk, L., Kundzewicz, Z. W. (1999): Dimensionality of Scandinavian river flow regimes. Hydrological Science Journal 44 (5):705-723. doi:https://doi.org/10.1080/ 02626669909492269

Laaha, G., Parajka, J., Viglione, A., Koffler, D., Haslinger, K., Schöner, W., Zehetgruber, J. Blöschl, G. (2016): A three-pillar approach to assessing climate impacts on low flows. Hydrology and Earth System Science 20:3967-3985. doi:https://doi.org/10.5194/hess-20-3967-2016 Laghari, A. N., Vanham, D., Rauch, W. (2012): To what extent does climate change result in a shift in Alpine hydrology? A case study in the Austrian Alps. Hydrological Science Journal 57:1:103-117. doi:https://doi.org/10.1080/ 02626667.2011.637040

Laizé, C. L. R., Acreman, M. C., Schneider, C. Dunbar, M.J., Houghton-Carr, H. A., Flörke, M. Hannah, D. M. (2014): Projected flow alteration and ecological risk for pan-European rivers. River Research and Applications 30 (3): 299-314 doi:https://doi.org/10.1002/rra.2645

Mader, H., Steidl, T., Wimmer, R. (1996): Abflußregime österreichischer Fließgewässer. Beitrag zu einer bundesweiten Fließgewässertypologie. In: BUJF (Hrsg). Monographien, Bd 82. Umweltbundesamt, Wien, S 192

Merz, R., Blöschl, G. (2005): Saisonalität von Niederschlag und Abfluss. In: BMLFUW (Hrsg). Hydrologischer Atlas Österreichs. Bundesminis terium für Land- und Forstwirtschaft, Umwelt und Wasserwirtschaft, Wien, S Karte 5.3

Milano, M., Reynard, E., Bosshard, N., Weingartner, R. (2015): Simulating future trends in hydrological regimes in Western Switzerland. Journal of Hydrology: Regional Studies 4 (B):748-761. doi:https://doi.org/10.1016/j.ejrh. 2015.10.010

Nilsson, C., Renöfält, B. M. (2008): Linking Flow Regimes and Water Quality in Rivers: a Challenge to Adaptive Catchment Management. Ecology and Society 13 (2): Art.18

Parajka, J., Kohnova, S., Bálint, G., Barbuc, M, Borga, M., Claps, P., Cheval, S., Dumitrescu, A. Gaume, E., Hlavcová, K., Merz, R., Pfaundler, M., Stancalie, G., Szolgay, J., Blöschl, G. (2010) Seasonal charactersitics of flood regimes across the Alpine-Carpathian range. Journal of Hydrology 394:78-89. doi:https://doi.org/10.1016/j. jhydrol.2010.05.015

Pardé, M. (1933): Fleuves et Rivieres. Armand Collin, Paris

Perkin, J. S., Bonner, T. H. (2011): Long-term changes in flow regime and fish assemblage composition in the guadalupe and san marcos rivers of texas. River Research and Applications 27:566-579. doi:https://doi.org/10.1002/ rra. 1373

Pfaundler, M., Dübendorfer, C., Zysset, A. (2011): Methoden zur Untersuchung und Beurteilung der Fliessgewässer. Hydrologie - Abflussregime Stufe F (flächendeckend). Bundesamt für Umwelt (BAFU), Bern

Poff, N. L., Allan, J. D., Bain, M. B., Karr, J. R., Prestegaard, K. L, Richter, B. D., Sparks, R. E. Stromberg, J. C. (1997): The natural flow regime A paradigm for river conservation and restoration. BioScience 47 (11):769-784. doi:https://doi. org/10.2307/1313099

Schneider, C., Laizé, C. L. R., Acreman, M. C. Flörke, M. (2013): How will climate change modify river flow regimes in Europe? Hydrology and Earth System Science 17:325-339. doi:https:// doi.org/10.5194/hess-17-325-2013

Stahl, K., Hisdal, H., Hannaford, J., Tallaksen, L. M., van Lanen, H. A. J., Sauquet, E., Demuth, S., Fendekova, M., Jódar, J. (2010): Streamflow trends in Europe: evidence from a dataset of near-natural catchments. Hydrology and Earth System Sciences 14:2367-2382. doi:https://doi. org/10.5194/hess- 14-2367-2010

Ständiges Sekretariat der Alpenkonvention (Hrsg) (2016): Die Alpen. Acht Staaten, ein Gebiet. Innsbruck

Stanzel, P., Nachtnebel, H. P. (2010): Mögliche Auswirkungen des Klimawandels auf den Wasserhaushalt und die Wasserkraftnutzung in Österreich. Österreichische Wasser- und Abfallwirtschaft 62 (9-10):180-187

Vanham, D., Rauch, W. (2009): Retreating snowpacks under climate change: implications for water resources management in the Austrian Alps. In: IAHS (Hrsg) Improving Integrated Surface and Groundwater Resources Management in a Vulnerable and Changing World. (Proc. of JS. 3 at the Joint IAHS \& IAH Convention). IAHS Publ. 330, Hyderabad, India, S 231-238

Weber, M., Braun, L, Mauser, W, Prasch, M. (2010): Contribution of rain,snow-and icemelt in the Upper Danube discharge today and in the future. Geografia Fisica e Dinamica Quaternaria $33: 221-230$

Wilson, D., Hannah, D. M., McGregor, G. R. (2013): A large-scale hydroclimatological perspective on Western European river flow regimes. Hydrology Research 44 (5):809-833. doi:https://doi.org/10.2166/nh.2012.201

Wrzesinski, D., Sobkowiak, L. (2018): Detection of changes in flow regime of rivers in Poland. J Hydrol Hydromech 66:55-64. doi:https://doi. org/10.1515/johh-2017-0045

Zampieri, M., Scoccimarro, E., Gualdi, S., Navarra, A. (2015): Observed shift towards earlier spring discharge in the main Alpine rivers. Science of the Total Environment 503-504:222232. doi:https://doi.org/10.1016/j.scitotenv. 2014.06.036 